\title{
ON SEQUENCES GENERIC IN THE SENSE OF PRIKRY
}

\author{
A. R. D. MATHIAS \\ (Received 15 September 1969; revised 22 August 1970) \\ Communicated by G. N. Crossley
}

I establish here a criterion for a sequence of ordinals to be generic over a transitive model of ZFC with respect to a notation of forcing first considered by Prikry in his Doctoral dissertation [2]. In Section 0 I review some notation, in Section $1 \mathrm{I}$ list some facts about measurable cardinals, and in Section 2, after giving Prikry's result, I state and prove mine.

Theorem 2.2 was proved during my brief stay at Monash University in Melbourne in June 1969. I thank Professor Crossley of that organisation for his hospitality. The paper was written in my sister's house in Pakistan.

\section{Notation}

In general I follow that of [1], but on Formalist grounds I use " $={ }_{d f}$ " to separate definiendum from definiens even where it is fashionable to write " $\Leftrightarrow_{d f}$ ".

Let $\kappa$ be an infinite initial ordinal. I use the letters $s, t, \cdots$ for finite subsets of $\kappa$, and $S, T, S^{\prime}, \cdots$ for infinite. 0 is the empty set and the first ordinal.

Definition 0.1. $|s|=_{d f} \max \{\alpha+1 \mid \alpha \in s\}$.

In particular, $|s|=0$ iff $s=0 ; s \neq 0 \rightarrow|s|=\beta+1$, for some $\beta$.

Definition 0.2. $s$ in $S={ }_{d f} \exists: \alpha<\kappa \quad s=\alpha \cap S$

(" $s$ is an initial segment of $S$ ").

Definition 0.3. $S \subseteq f_{f^{\prime}} T=_{d f} \exists s:$ in $S \quad S-|s| \subseteq T$

(" $S$ is, apart from finitely many elements, a subset of $T$ ").

Let $\underset{=}{F}$ be a set of infinite subsets of $\kappa$.

Definition 0.4. $P_{\underline{\underline{F}}}={ }_{d f}\{\langle s, S\rangle|| s \mid \leqq \min S \wedge S \in \underline{\underline{F}}\}$.

I use letters $p, q \cdots$ for elements of $P_{\underline{F}}$.

The following partial ordering will be important:

DEFINITION 0.5 (Prikry). $\leqq=_{d f}\{\langle\langle s, S\rangle,\langle t, T\rangle\rangle \mid S \subseteq T \wedge t \subseteq s \wedge s-t \subseteq T\}$. 
DEFINITION 0.6. $\mathbb{P}_{\underline{\underline{F}}}={ }_{d f}\left\langle P_{\underline{\underline{F}}}, \leqq \cap P_{\underline{\underline{F}}}^{2}\right\rangle$.

Let

$$
\Delta \subseteq P_{\underline{F}} \text {. }
$$

Definition 0.7. $\Delta$ is dense in $\mathbb{P}_{\underline{\underline{F}}}={ }_{d f} \forall p: \in P_{\underline{\underline{F}}} \exists q: \in \Delta q \leqq p$.

Definition 0.8. $\Delta$ is $\leqq$-closed in $\mathbb{P}_{\underline{F}}={ }_{d f} \forall p: \in \Delta \forall q: \in P_{F}(q \leqq p \rightarrow q \in \Delta)$.

Let $M$ be a transitive $\varepsilon$-model of $Z F+A C$; let $\kappa$ and $F$ be elements of $M$. Then $\mathbb{P}_{\underline{\underline{F}}} \in M$. In the sequel, $M$ may be taken to be a set or a proper class: it is left to the reader to interpret the theorems and arguments as theorem and proof schemata of $Z F$ when appropriate.

Let $a \subseteq \kappa, a$ of order type $\omega$.

Definition 0.9. $F_{a}=\left\{\langle s, S\rangle \mid S \subseteq a \subseteq s \cup S \wedge\langle s, S\rangle \in \mathbb{P}_{\underline{\underline{F}}}\right\}$.

Definition 0.10. $a$ is $\mathbb{P}_{\underline{\underline{F}}}$-generic over $M=_{d f}$

$$
\begin{aligned}
& \forall \Delta: \in M\left(\Delta \text { dense and } \leqq \text {-closed } \rightarrow \Delta \cap F_{a} \neq 0\right) \wedge \\
& \forall p, q: \in F_{a} \exists q^{\prime}: \in P_{\underline{F}}\left(q^{\prime} \leqq p \wedge q^{\prime} \leqq q\right) \wedge \\
& \forall p: \quad \in F_{a} \forall q: \in P_{F}\left(p \leqq q \rightarrow q \in F_{a}\right) .
\end{aligned}
$$

Remark. The above is equivalent in $Z F$ to all other customary definitions of genericity with respect to a partial ordering and a model of $Z F$.

\section{Measurable cardinals}

DefINITION 1.1. $\underline{\underline{U}}$ is a two-valued measure on $\kappa={ }_{\mathbf{d} f} \underset{\underline{U}}{U}$ is a non-principal ultrafilter on $\kappa$ and whenever $\lambda\left\langle\kappa\right.$ and $\left\langle A_{i}\right| i\langle\lambda\rangle$ is a sequence of elements of $\underline{\underline{U}}, \bigcap_{i<\lambda} A_{i} \in \underline{\underline{U}}$.

Definition 1.2. Let $A \subseteq \kappa . \mid A]^{n}=_{d f}\{s \subseteq A \mid \overline{\bar{s}}=n\}$.

Note that $0 \in[A]^{<\omega}$.

$$
\mid A]^{<\omega}={ }_{\mathrm{d} f} \cup\left\{[A]^{n} \mid n<\omega\right\} .
$$

DEFINITION 1.3. $\underset{=}{U}$ is a normal measure on $\kappa={ }_{d f} \underline{\underline{U}}$ is a two-valued measure on $\kappa$ and whenever $\left\langle A_{t} \mid t \in[\kappa]^{<\omega}\right\rangle$ is a family of elements of $\underline{\underline{U}}$ indexed by the finite subsets of $\kappa$, there is a $B \in \underset{=}{U}$ such that

$$
\forall t: \in[\kappa]^{<\omega} B-|t| \subseteq A_{t} .
$$

The following lemma verifies that that definition is equivalent to the usual definitions of normal measure.

LEMMA 1.4. Let $U$ be a two-valued measure on $\kappa$. $U$ is normal if and only if for any sequence $\left\langle C_{\alpha}\right| \alpha\langle\kappa\rangle$ of elements of $\underline{\underline{U}}$ such that $\forall \alpha:<\kappa \quad C_{\alpha}=$ $\cap\left\{C_{\beta+1} \mid \beta<\alpha\right\},\left\{\alpha \mid \alpha \in C_{\alpha}\right\} \in \underset{=}{U}$. 
PRoor. Suppose $\underline{=}$ normal and let $\left\langle C_{\alpha} \mid \alpha<\kappa\right\rangle$ be a sequence of elements of $\underline{\underline{U}}$ such that

$$
\forall \alpha:<\kappa C_{\alpha}=\cap\left\{C_{\beta+1} \mid \beta<\alpha\right\} .
$$

Let $A_{s}=\cap\left\{C_{v+1} \mid v \in s\right\}$ if $\left.s \in \mid \kappa\right]^{<\omega}$ and $s \neq 0$, and let $A_{0}=C_{0}$. Let $B \in U$ be such that $B-|s| \subseteq A_{s}$, and let $v \in B$. If $v=0$, then $v \in A_{0}$ and so $v \in C_{0}^{=}$. If $v=\alpha+1$, then $v \in A_{\{\alpha\}}$ and so $v \in C_{\alpha+1}=C_{v}$. If $v$ is a limit ordinal, let $\beta<v$ : then $v \in A_{\{\beta\}}$ and so $v \in C_{\beta+1}$; so

$$
v \in \cap\left\{C_{\beta+1} \mid \beta<v\right\}
$$

which is $C_{v}$. Thus $B \subseteq\left\{v \mid v \in C_{v}\right\}$ which is therefore in $U$.

Contrariwise, if $\left\langle A_{s} \mid s \in[\kappa]^{\langle\omega}\right\rangle$ is a family of elements of a two-valued measure $U$ which satisfies the hypothesis of the lemma on sequences $\left\langle C_{\alpha} \mid \alpha<\kappa\right\rangle$, define

$$
C_{\alpha}=\cap\left\{A_{s}|| s \mid \leqq \alpha\right\} \text {. }
$$

Then $\forall \alpha C_{\alpha}=\cap\left\{C_{\beta+1} \mid \beta<\alpha\right\}$ as $|s|$ is never a limit ordinal, and each $C_{\alpha}$ is in $\underline{\underline{U}}$ as

$$
\alpha<\kappa \rightarrow\{s|| s \mid \leqq \alpha\}
$$

has cardinality $<\kappa$, and so, writing $B=\left\{\alpha \mid \alpha \in C_{\alpha}\right\}, B \in \underline{\underline{U}}$. If $s=0, B-0=B$, and

$$
\forall \alpha: \in B \alpha \in C_{0}=A_{0},
$$

so $B-|0| \subseteq A_{0}$. If $s \neq 0$, let $\alpha=\max s$. Let $\beta \in B-|s|$ : then $\beta \in C \subseteq A_{s}$ as $|s|=\alpha+1 \leqq \beta$; so $B-|s| \subseteq A_{s}$.

THeOREM 1.5 (Scott See for example Solovay [3].). $(Z F+A C)$ If $\kappa$ has a two-valued measure, it has a normal measure.

TheOREM 1.6 (Rowbottom). $(Z F+A C)$ Let $U$ be a normal measure on $\kappa$; let $\lambda<\kappa$ and $f: \mid \kappa]^{<\omega} \rightarrow \lambda$. Then $\exists A: \in \underset{=}{U} \forall n: \stackrel{=}{<} \omega \forall x, y: \in[A]^{n} f(x)=f(y)$.

Such an $A$ is said to be homogeneous for $f$.

I sketch a proof of Rowbottom's theorem. You show first by induction on $n$ that

$$
\forall f^{\prime}\left(\left(f^{\prime}:[K]^{n} \rightarrow \lambda\right) \rightarrow \exists A: \in \underline{\underline{U}} \forall x, y: \in[A]^{n} f^{\prime}(x)=f^{\prime}(y)\right) .
$$

For $n=0(\dagger)$ is trivial, and for $n=1$ it follows from the property that

$$
\forall \alpha:<\lambda C_{\alpha} \in \underline{\underline{U}} \rightarrow \cap\left\{C_{\alpha} \mid \alpha<\lambda\right\} \in \underline{\underline{U}} .
$$

Suppose true for $n=k$, and let $f^{\prime}:[\kappa]^{k+1} \rightarrow \lambda$. Then for each $s \in[\kappa]^{k}$ there is an $A_{s} \in U$ such that $f^{\prime}$ is constant on $\left\{s \cup\{\alpha\} \mid \alpha \in A_{s}\right\}$ and $A_{s} \subseteq \kappa-|s|$. Let $g(s)$ be that constant value of $f^{\prime}$. Let $A_{s}=\kappa$ if $s \notin[\kappa]^{k}$. Let $B \in \underline{\underline{U}}$ be such that $\forall s B-|s| \subseteq A_{s}$. Let $C \in \underset{=}{U}$ be such that 


$$
\forall s, t: \in[C]^{k} g(s)=g(t) \text {. }
$$

(Such a $C$ exists by the induction hypothesis.) Let $A=B \cap C$. Then $f^{\prime}$ is constant on $[A]^{k+1}$.

To prove the theorem, pick for each $n$ an $A^{(n)} \in \underline{\underline{U}}$ such that $f$ is constant on $\left[A^{(n)}\right]^{n}$, and let

$$
A=\cap\left\{A^{(n)} \mid n<\omega\right\} .
$$

\section{Prikry sequences}

THEOREM 2.1 (Prikry). Let $M$ be a transitive model of $Z F+A C$; let $\kappa \in M$, and let $U \in M$ be in $M$ a normal measure on $\kappa$. Let a be a subset of $\kappa$ of order type $\omega$, and suppose that a is $\mathbb{P}_{U}$ generic over $M$. Then every cardinal in $M$ is a cardinal in $M[a] ; a$ is cofinal in $\kappa$, and so $\kappa$ is of cofinality $\omega$ in $M[a]$; and if $\lambda<\kappa, b \subseteq \lambda$ and $b \in M[a]$, then $b \in M$.

The principal result of the paper is now stated.

THEOREM 2.2. Let $M, \kappa, U$ be as in 2.1. Let $a \subseteq \kappa$ be of order type $\omega$. Then a is $\mathbb{P}_{\underline{\underline{v}}}$-generic over $M$ if and only if

$$
\forall A: \in \underset{=}{U} a \subseteq_{f} A .
$$

Here $a \subseteq_{f} A$ is as defined in 0.3 .

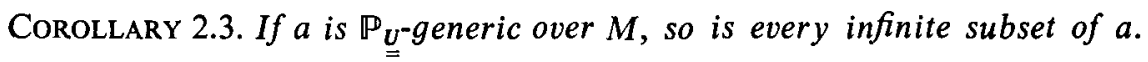

The proof of Theorem 2.2 uses Theorem 1.6, as did Prikry's proof of 2.1. For the time being $I$ argue in the theory $Z F+A C$ with the assumption that $\underline{\underline{U}}$ is a normal measure on $\kappa$.

Definition 2.4. Let $\Delta$ be a dense, $\leqq$-closed subset of $P_{U} . s$ a (finite) subset of $\kappa . T$ captures $\langle s, \Delta\rangle={ }_{d f}|s| \leqq \min T \wedge \exists n:\left\langle\omega\left(\bar{\forall} t\left(t \in[T]^{n} \rightarrow\langle s \cup t\right.\right.\right.$, $T-|t|\rangle \in \Delta))$.

LeMMA 2.5. $(Z F+A C)$ Let $\Delta$ be a dense $\leqq$-closed subset of $P_{U}$.

$$
\forall s: \subseteq \kappa \exists T: \in \underline{\underline{U}}(T \text { captures }\langle s, \Delta\rangle)
$$

Proof. Let $\Delta, s$ be given. To each $t \subseteq \kappa-|s|$ pick $A_{t} \in \underset{\underline{U}}{\text { such that }}$

$$
(\exists A: \in \underset{=}{U}\langle s \cup t, A\rangle \in \Delta) \rightarrow\left\langle s \cup t, A_{t}\right\rangle \in \Delta .
$$

Let $A_{t}=\kappa$ if $t \nsubseteq \kappa-|s|$. By the normality of $\underline{\underline{U}}$ there is a $B^{\prime} \in \underline{\underline{U}}$ such that

$$
\forall t: \in[\kappa]^{<\omega} B^{\prime}-|t| \subseteq A_{t} \text { : }
$$

let $B=B^{\prime} \cap(\kappa-|s|)$. Then $B \in \underline{\underline{U}}$ and

$\left(^{*}\right) \forall t: \subseteq B((\exists A: \in \underline{\underline{U}}\langle s \cup t, A\rangle \in \Delta) \rightarrow\langle s \cup t, B-|t|>\in \Delta)$, 
for if $t \cup B$ and $\exists A: \in U\langle s \cup t, A\rangle \in \Delta$ then $\left\langle s \cup t, A_{t}\right\rangle \in \Delta ; B-|t| \subseteq A_{t}$; and so $\langle s \times t, B-|t| \in \Delta$ as $\Delta$ is $\leqq$-closed.

Define a map $f:[\kappa]^{<\omega} \rightarrow 3$ by

$$
\begin{aligned}
& f(t)=0 \text { if } t \neq B ; \\
& f(t)=1 \text { if } t \subseteq B \text { and }\langle s \cup t, B-|t|\rangle \in \Delta ; \\
& f(t)=2 \text { if } t \subseteq B \text { and }\langle s \cup t, B-|t|\rangle \notin \Delta .
\end{aligned}
$$

Let $C \in \underline{\underline{U}}$ be homogeneous for $f$, and let $T=C \cap B$. Then $T \in \underline{\underline{U}}$.

As $\Delta$ is dense,

$$
\exists t: \subseteq T \exists T^{\prime} \subseteq T\left(|t| \leqq \min T^{\prime} \text { and }\left\langle s \cup t, T^{\prime}\right\rangle \in \Delta\right) .
$$

Fix such a $t$. Let $n=\overline{\bar{t}}$. As $T \subseteq B$, by $\left(^{*}\right)\langle s \cup t, B-|t|\rangle \in \Delta$, and so $f(t)=1$. That $T$ captures $\langle s, \Delta\rangle$ remains to be seen.

Let $t^{\prime} \subseteq T$ and $\bar{t}^{\prime}=n$. As $T$ is homogeneous for $f, f\left(t^{\prime}\right)=f(t)=1$, so

$$
\left\langle s \cup t^{\prime}, B-\left|t^{\prime}\right|\right\rangle \in \Delta
$$

as $\Delta$ is $\leqq$-closed and $T-\left|t^{\prime}\right| \subseteq B-\left|t^{\prime}\right|$,

$$
\left\langle s \cup t^{\prime}, T-\left|t^{\prime}\right|\right\rangle \in \Delta \text {. }
$$

Proof of Theorem 2.2. Suppose a $\mathbb{P}_{\underline{\underline{v}}}$-generic over $M$, and let $A \in \underset{\equiv}{U}$. Let

$$
\Delta=[\langle s, S\rangle \mid s \in \underline{\underline{U}} \wedge S \subseteq A\} .
$$

$\Delta$ is dense, $\leqq$-closed and in $M$, so there is an

$$
\langle s, S\rangle \in \Delta \cap F_{a}: s \subseteq a \subseteq s \cup S ;
$$

so $a \subseteq_{f} S \subseteq A$ and hence $a \subseteq_{f} A$.

Now suppose that $\forall A: \in \bigcup_{=} a \subseteq_{f} A$ and let

$$
F_{a}=\{\langle s, S\rangle \mid S \in \underline{\underline{U}} \wedge s \subseteq a \subseteq s \cup S\},
$$

as in Definition 0.9. It must now be shown that $F_{a}$ has the three properties listed in Definition 0.10.

(iii) Let $\langle s, S\rangle \in F_{a}$, and $\langle s, S\rangle \leqq\left\langle s^{\prime}, S^{\prime}\right\rangle \in P_{\underline{\underline{U}}}$. Then

$$
s^{\prime} \subseteq s \subseteq a \subseteq s \cup S \subseteq s^{\prime} \cup S^{\prime},
$$

so $\left\langle s^{\prime}, S^{\prime}\right\rangle \in F_{a}$.

(ii) Let $\langle s, S\rangle$ and $\left\langle s^{\prime}, S^{\prime}\right\rangle \in F_{a} . s \cup s^{\prime} \subseteq a$ and

$$
a \subseteq(s \cup S) \cap\left(s^{\prime} \cup S^{\prime}\right),
$$


$\left\langle s \cup s^{\prime}, S \cap S^{\prime}\right\rangle \leqq\langle s, S\rangle,\left\langle s \cup s^{\prime}, S \cap S^{\prime}\right\rangle \leqq\left\langle s^{\prime}, S^{\prime}\right\rangle$, and

$$
\left\langle s \cup s^{\prime}, S \cap S^{\prime}\right\rangle \in P_{\underline{\underline{U}}} .
$$

(i) Let $\Delta \in M, \Delta$ dense and $\leqq$-closed. Working in $M$ and using Lemma 2.5, pick for each $s \subseteq \kappa$ a $T_{s} \in U$ that captures $\langle s, \Delta\rangle$. There is a $B \in U$ such that $\forall s B-|s| \subseteq T_{s} . a \subseteq{ }_{f} B$; so let $\sin a$ be such that $a-|s| \subseteq B$. Then $a-|s| \subseteq T_{s}$; as $T_{s}$ captures $\langle s, \Delta\rangle$, there is an $n$ such that in $M$,

$$
\left.t \in \mid T_{s}\right]^{n} \rightarrow\left\langle s \cup t, T_{s}-|t|\right\rangle \in \Delta .
$$

Let $t^{\prime}$ be the set of the first $n$ elements of $a-|s|$. Then $\left\langle s \cup t^{\prime}, T_{s}-\left|t^{\prime}\right|\right\rangle \in \Delta \cap F_{a}$.

Finally let me derive the lemma used by Prikry in his proof of Theorem 2.1 from Lemma 2.5, to which it is a kin.

LEMMA 2.6 (Prikry). Let $\mathfrak{A}$ be a sentence of the language of forcing and $\langle s, S\rangle \in P_{\underline{\underline{U}}}$. Then

$$
\exists S^{\prime} \subseteq S\left(S^{\prime} \in U \wedge\left(\left\langle s, S^{\prime}\right\rangle\left|\vdash \mathfrak{A} \vee\left\langle s, S^{\prime}\right\rangle\right| \vdash \neg \mathfrak{A}\right)\right) .
$$

Proof. Let $\Delta=\{\langle t, T\rangle|\langle t, T\rangle| \vdash \mathfrak{A} \vee\langle t, T\rangle ; \vdash \neg \mathfrak{A}\}$. As $\Delta$ is dense and $\leqq$-closed there are by Lemma 2.5 an $S^{\prime \prime} \subseteq S$ and an $n \in \omega$ such that

$$
\forall t: \in\left[\left.S^{\prime \prime}\right|^{n}\left\langle s \cup t, S^{\prime \prime}-|t|\right\rangle \in \Delta .\right.
$$

Define $f:\left[S^{\prime \prime}\right]^{n} \rightarrow 2$ by

$$
\begin{aligned}
f(t) & =0 \text { if }\left\langle s \cup t, S^{\prime \prime}-|t|\right\rangle \mid \vdash \mathfrak{A} \\
& =1 \text { if }\left\langle s \cup t, S^{\prime \prime}-|t|\right\rangle \mid \vdash \neg \mathfrak{A} .
\end{aligned}
$$

Let $S^{\prime} \subseteq S^{\prime \prime}$ be homogeneous for $f$. If neither $\left\langle s, S^{\prime}\right\rangle \mid \vdash \mathfrak{A}$ nor $\left\langle s, S^{\prime}\right\rangle \mid \vdash \neg \mathfrak{A}$, there are $s^{\prime}, s^{\prime \prime}, T^{\prime}, T^{\prime \prime} \subseteq S^{\prime}$ with

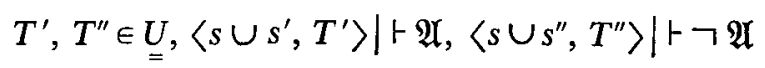

and, it may be assumed, $\min \left\{\bar{s}^{\prime}, \overline{\bar{s}}^{\prime \prime}\right\} \geqq n$. Let $t^{\prime}$ be the first $n$ element of $s^{\prime}$ and $t^{\prime \prime}$ of $s^{\prime \prime}$. Then $f\left(t^{\prime}\right)=0$ and $f\left(t^{\prime \prime}\right)=1$ (for $S^{\prime \prime}$ captures $\langle s, \Delta\rangle$ ), which contradicts the homogeneity of $S^{\prime}$.

\section{References}

[1] R. B. Jensen, Modelle der Mengenlehre (Springer-Verlag, Lecture Notes Series, 1968).

[2] K. L. Prikry, Doctoral dissertation (Berkeley, 1968). Published as 'Changing measurable into accessible cardinals' in Dissertationes Math. (Rozprawy Mat.) 68 (1969).

[3] R. M. Solovay, 'Real-valued measurable cardinals,' in the Proceedings of the 1967 Summer Institute in Set Theory held at Los Angeles.

Peterhouse, Cambridge

England 